\title{
The Significance of Transcriptomic Signatures in the Multifocal Papillary Thyroid Carcinoma: Two mRNA Expression Patterns with Distinctive Clinical Behavior from The Cancer Genome Atlas (TCGA) Database
}

\author{
Yea Eun Kang ${ }^{1 *}$, Boyoung Hwang ${ }^{1 *}$, Ju Hee Lee ${ }^{1}$, Minho Shong ${ }^{1}$, \\ Hyon-Seung $\mathrm{Yi}^{1}$, Bon Seok $\mathrm{Koo}^{2}$ and Dong Jin Lee ${ }^{3}$
}

Division of Endocrinology and Metabolism, Department of Internal Medicine, Chungnam National University College of Medicine ${ }^{1}$, Department of Otolaryngology-Head and Neck Surgery, Chungnam National University College of Medicine', Daejeon, Department of Otolaryngology-Head and Neck Surgery, Hallym University College of Medicine, Seoul, Korea

\begin{abstract}
Background and Objectives: The association between multifocal papillary thyroid carcinoma (PTC) and tumor aggressiveness remains controversial. The aim of study is to evaluate molecular subtypes of multifocal PTCs using multiplatform genomic analysis. Materials and Methods: Statistical analysis and genomic analysis were performed for gene expression data and clinical data of multifocal PTCs in The Cancer Genome Atlas data. Clinicopathologic findings, recurrence-free survival (RFS), copy number alteration and somatic mutation status in patients in relation to molecular subtypes were analyzed. Results: Multiplatform genomic analysis revealed that multifocal PTCs $(n=226)$ were divided into two distinct molecular subgroups. Participants in cluster 2 showed significantly increased risk of extrathyroidal extension, lymph node metastasis, and BRAF ${ }^{\mathrm{V} 600 \mathrm{E}}$ mutation compared to patients in cluster 1. To exclude the effect of BRAF mutation and RAS mutation on tumor aggressiveness, we compared clinical parameters between two clusters in patients without BRAF or RAS mutation. Cluster 2 showed significantly higher risk of lymph node metastasis compared to cluster 1. Conclusion: Multifocal PTC has two distinct molecular subtypes with distinctive clinical behaviors. Our data suggested the clinical implications of the transcriptomic signature to predict clinical outcomes of multifocal PTC.
\end{abstract}

Key Words: Thyroid neoplasm, Gene expression profiling, Genomics

\section{Introduction}

Papillary thyroid cancer (PTC) is one of the most common endocrine cancers. Its incidence has been rising steadily. ${ }^{1)}$ PTC presents variously, ranging from a single solitary nodule to multiple co-existing nodules arising from separate foci in the thyroid gland. ${ }^{2,3)}$ Multifocal pattern in PTC has a high rate ranging from $24 \%$ to $46 \%$, among which $50-70 \%$ are bilateral. ${ }^{4-6)}$

The correlation between multifocality and clinical aggressiveness of PTC remains controversial. Many studies reported multifocality is associated with progression of PTC. ${ }^{7-9)}$ However, some of recent studies

Received May 14, 2019 / Revised lst April 1, 2020, 2nd May 4, 2020 / Accepted May 8, 2020

Correspondence: Dong Jin Lee, MD, PhD, Department of Otolaryngology-Head and Neck Surgery, Hallym University College of Medicine, l Singil-ro, Yeongdeungpo-gu, Seoul 0744l, Korea

Tel: 82-2-829-5217, Fax: 82-2-842-5217, E-mail: leesurgery@gmail.com

*Authors contribute equally. 
reported opposite result. Singhal et al. ${ }^{10)}$ reported multifocal PTC showed less extrathyroidal extension (ETE) than solitary nodule. Konturek et al. ${ }^{11)}$ reported that multifocal PTC showed less distant metastasis than solitary nodule. Ahn et al. ${ }^{12)}$ reported that odds ratio of lymph node metastasis didn't show significant difference between multifocal and solitary PTC. ${ }^{12)}$ In this reason, classification of molecular subtyping of multifocal PTC is absolutely needed to predict clinical behavior of multifocal PTC.

The Cancer Genome Atlas (TCGA) research network group has analyzed multiple genomic platform data of cancers, including PTCs data. ${ }^{13)}$ They share valuable data at publicly available websites with diverse clinical information, including mRNA expression using RNA sequence, mutation profile, copy number alteration, micro-RNA expression, methylation profile, and exon sequencing data. Molecular classification of cancer type using these multiplatform data makes it possible to predict clinical behavior and give appropriate treatment, especially for aggressive tumor subtypes.

In this study, using a large set of TCGA mRNA expression data, we classified multifocal PTCs into two subgroups with distinct molecular patterns and different clinical behaviors. Differences in involved pathways between these subgroups were determined using an integrated gene-level approach based on gene expression data, copy number alteration data and somatic mutation data.

\section{Materials and Methods}

\section{Genomic and Clinical Data Sets}

All genomic data of PTC from TCGA project were obtained from TCGA data portal (https://tcga-data. nci.nih.gov) and cancer browser (https://genomecancer.ucsc.edu). Effective samples included both clinical pathological information, including presence of other thyroid disease. Patients with multifocal PTC $(n=226)$ of total 505 participants were enrolled in our study. 80 patients were diagnosed at bilateral PTC, and others represented unilateral PTC. All parameters including age, gender, tumor size, extrathyroidal ex- tension, TNM stage, presence of thyroid disease, and BRAF mutation state in 226 patients were analyzed. Minimal ETE is defined as tumor cells extending to the sternothyroid muscle or perithyroidal soft tissue, and this is a departure from the 7th edition of the AJCC staging, in which ETE was distributed across T3, T4a, and T4b categories to reflect minimal ETE (including microscopic ETE and macroscopic ETE into strap muscles). Moderate/Advanced ETE, extensive ETE, is defined as tumor of any size that has grown beyond the thyroid capsule to invade subcutaneous soft tissues, larynx, trachea, esophagus, recurrent laryngeal nerve, or major vessels by the tumor. Gene expression data from mRNA-seq, copy number alteration data, somatic mutation data, and clinical parameters in patients with multifocal PTC $(n=226)$ were analyzed. Clinical data included age, gender, tumor size, extrathyroidal extension, TNM stage (AJCC $8^{\text {th }}$ ), RAS mutation state, and BRAF mutation state (Supplementary Data 1).

\section{Analysis of Gene Expression Data and Supervised Clustering}

All the samples used to test the gene expression are from human thyroid tissue. Effective samples included both clinical and pathological information, and the expression levels of the whole genome (total of 20,531 genes). The expression levels of genes were measured as the reads per kilobase per million reads. Gene expression data were gene-median centered using BRB-ArrayTools software program (http://linus. nci.nih.gov/BRB-ArrayTools.html). ${ }^{14)}$ Gene variability was then computed using median absolution deviation. A total of 3134 most variable genes were selected. A heatmap was generated using Cluster and TreeView software programs. ${ }^{15)}$

\section{Analysis of Copy Number Alteration Data}

Copy number alteration analysis was performed using Cluster and TreeView software programs.

\section{Analysis of Somatic Mutation Data}

Somatic mutation analysis was performed using oncoprint at Cbioportal website (http://cbioportal.org). 


\section{Selection of Specific Gene Signature in Each Group}

Genes with a fold change greater than 2 (the absolute value of $\log 2$ fold change greater than 1) and an adjust $p$-value less than 0.05 were considered as differentially expressed and were selected genes in groups.

\section{Significant Canonical Signaling Pathways Enriched in Each Subgroup}

Pathway analysis was carried using Ingenuity Pathways Analysis (Ingenuity, Redwood City, CA, USA). Genes associated with canonical pathway in Ingenuity Pathways Knowledge Base were analyzed. Significance of association between differently expressed genes of each group and the canonical pathway was measured using Fischer's exact test at $p<0.001$.

\section{Statistical Analysis}

The association of molecular subtype with each clinical variable was evaluated using Chi-square test. Recurrence free survival (RFS) results between two groups were compared using Kaplan Meier plot and log rank test. Significant difference was considered at $p<0.05$. All statistical analyses were conducted in $R$ language environment (http://www.r-project.org) and SPSS statistics version 22.0 (IBM Corp., Armonk, NY, USA).

\section{Results}

\section{Unsupervised Hierarchical Clustering in Multifocal Papillary Thyroid Carcinomas}

To find statistically significant gene expression subtypes in multifocal PTCs $(n=226)$, hierarchical clustering in an unsupervised and unbiased manner was performed using cluster 3.0. program. Results of hierarchical clustering analysis revealed two molecular subtypes with distinct gene expression profiles (Fig. 1). Red and green colors in the heatmap indicated relative high and low expression, respectively.

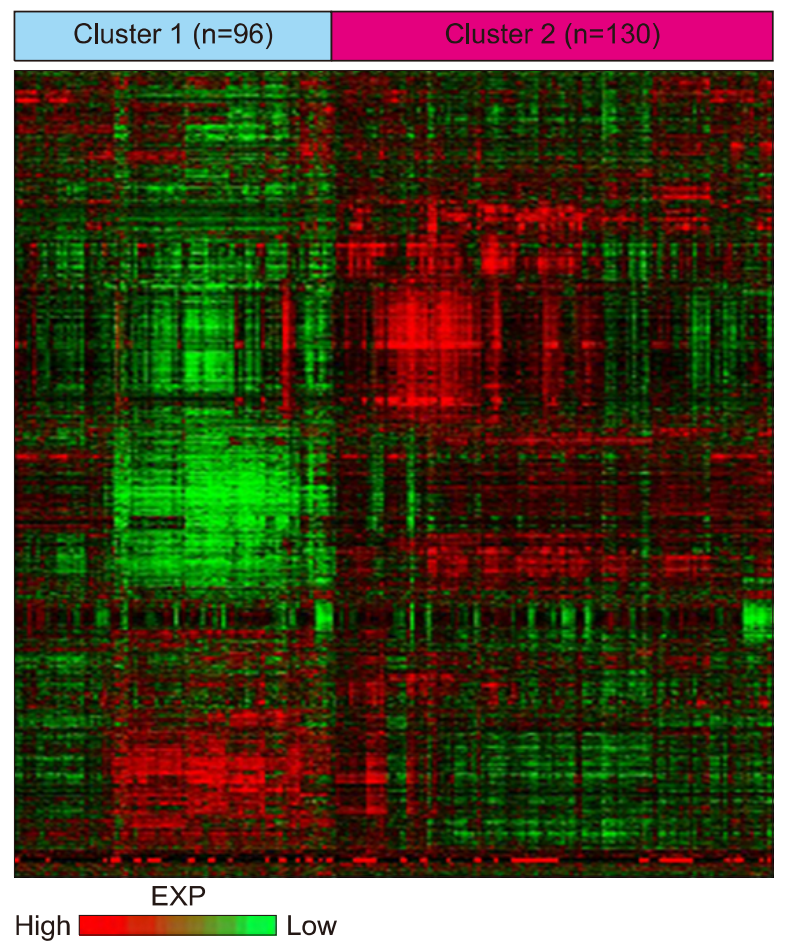

Fig. 1. Unsupervised clustering of multifocal papillary thyroid carcinomas (PTCs) revealing two distinct molecular subtypes. A hierarchical clustering of gene expression data from 226 multifocal PTC cases in The Cancer Genome Atlas (TCGA) data. Gene expression levels that were at least 2-fold different in at least 50 cases relative to the median value across cases were selected for hierarchical clustering analysis (3134 gene features). Data are given in matrix format, in which rows represent individual genes and columns represent each patient. Each cell in the matrix represents expression level of a gene feature in an individual pattern. Red and green colors in cell indicate relative high and low expression, respectively, as indicated in the scale bar.

\section{Comparison of Copy Number Alterations Data between Cluster 1 and Cluster 2}

As shown in Fig. 2, copy number alterations of cluster 2 were almost none when compared to cluster 1. Cluster 1 multifocal PTCs had some CPN alterations such as chromosome 15,16,17,18,19 amplification and chromosome 20,21 deletion, but cluster 2 multifocal PTCs had chromosome 1q amplification and chromosome $6 q$ deletion.

\section{Comparison of Somatic Mutation Data between Cluster 1 and Cluster 2}

Since previous study suggested the importance of 


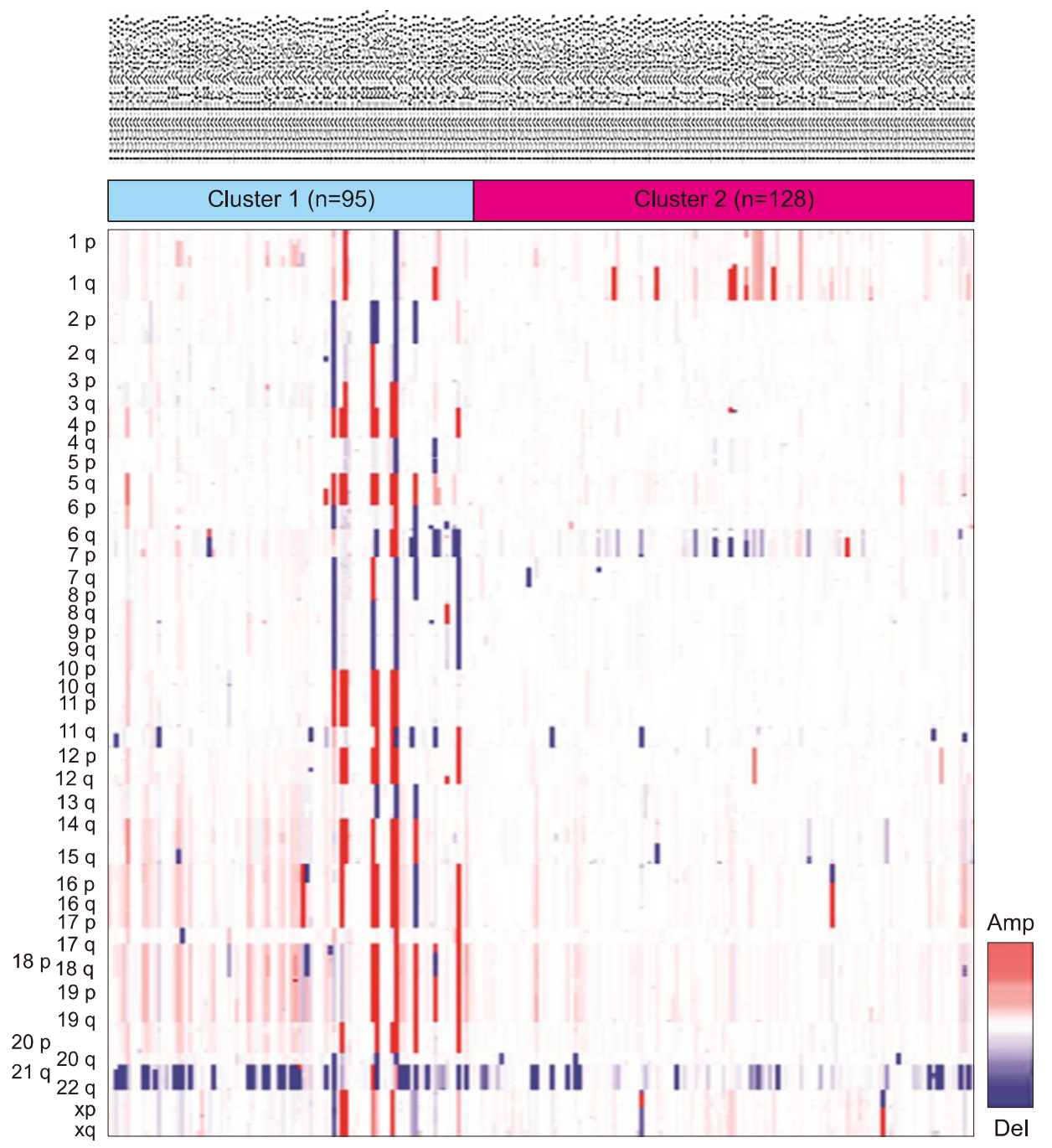

Fig. 2. Copy number (CPN) alteration according to each cluster. The amount of CPN variation of PTC is small $(-0.2$ to +0.1$)$ when compared to other cancer type. Multifocal PTCs in cluster 1 showed frequent CPN alteration when compared to cluster 2. both $\mathrm{BRAF}^{\mathrm{V} 600 \mathrm{E}}$ mutation status and RAS score to predict tumor aggressiveness, comparison of somatic mutation data between cluster 1 and cluster 2 were performed (Supplementary Fig. 1). We hypothesized that there was another molecular pathway related to tumor progression in multifocal PTC and the presence of well-known somatic mutation. Interestingly, cluster 2 had more frequent BRAF ${ }^{\mathrm{V} 600 \mathrm{E}}$ mutation $(n=85 / 130$, $65.4 \%$ ) compared to cluster 1. However, BRAF ${ }^{\mathrm{V} 600 \mathrm{E}}$ mutation was also detected in some patients in cluster $1(n=21 / 96,21.9 \%)$. Moreover, RAS mutation were detected only cluster 1 (HRAS mutation=7, NRAS mutation=13, and KRAS mutation=2) (Supplementary Fig. 1).

\section{Comparison of Clinicopathologic Parameters between Cluster 1 and Cluster 2}

To determine the importance of our molecular sub- types classification using integrated genomic analysis, we compared clinicopathologic parameters between cluster 1 and cluster 2 using Chi-square test and paired t test (Table 1). There was no significant difference in age, sex, or T stage between cluster 1 and cluster 2. Although tumor size was significantly more increased in cluster 1 compared to that in cluster 2 , the status of extra-thyroid extension, lymph node metastasis, and presence of BRAF ${ }^{\mathrm{V} 600 \mathrm{E}}$ mutation was less frequent in cluster 1 compared to that in cluster 2. In analysis of thyroid disease status between the two groups, nodular hyperplasia was significantly more increased in cluster 1 compared to that in cluster 2 . Since several groups identified the relationship of nodular hyperplasia or lymphocytic thyroiditis with tumor aggressiveness, ${ }^{16-18)}$ we investigated the relationship of thyroid disease with clusters. However, there was 
Table 1. Case distribution according to cluster 1 and cluster 2

\begin{tabular}{|c|c|c|c|}
\hline & \multicolumn{2}{|c|}{ Cluster } & \multirow{2}{*}{$p$-value } \\
\hline & Cluster $1 \quad(n=96)$ & Cluster $2(n=130)$ & \\
\hline Age & & & 0.59 \\
\hline Mean & $48.7(14.3)$ & $47.2(10.5)$ & \\
\hline Gender & & & 0.21 \\
\hline Male & 27 & 48 & \\
\hline Female & 69 & 82 & \\
\hline Size $(\mathrm{cm})$ & & & 0.04 \\
\hline Mean diameter & $1.81(0.9)$ & $1.51(1.0)$ & \\
\hline ETE & & & $<0.0001$ \\
\hline None & 80 & 75 & \\
\hline Minimal & 16 & 50 & \\
\hline Moderate/Advanced & 0 & 5 & \\
\hline T stage & & & 0.090 \\
\hline $\mathrm{T} 1$ & 66 & 102 & \\
\hline $\mathrm{T} 2$ & 26 & 20 & \\
\hline T3 & 4 & 8 & \\
\hline $\mathrm{N}$ stage & & & $<0.0001$ \\
\hline NO & 71 & 56 & \\
\hline $\mathrm{N} 1 \mathrm{a}$ & 11 & 52 & \\
\hline N1b & 12 & 36 & \\
\hline Stage & & & 0.092 \\
\hline Stage | & 83 & 99 & \\
\hline Stage $\|$ & 11 & 25 & \\
\hline Stage III & 1 & 6 & \\
\hline Stage IV & 1 & 0 & \\
\hline Unilateral/Bilateral & & & 0.259 \\
\hline Unilateral & 67 & 79 & \\
\hline Bilateral & 29 & 51 & \\
\hline Thyroid disease & & & 0.008 \\
\hline None & 51 & 90 & \\
\hline Lymphocytic thyroiditis & 9 & 17 & \\
\hline Nodular hyperplasia & 26 & 15 & \\
\hline Lymphocytic thyroiditis + nodular hyperplasia & 8 & 8 & \\
\hline BRAF & & & $<0.0001$ \\
\hline Wild type & 75 & 45 & \\
\hline V600E mutated & 21 & 85 & \\
\hline RAS & & & $<0.0001$ \\
\hline Wild type & 76 & 130 & \\
\hline HRAS mutation & 7 & 0 & \\
\hline NRAS mutation & 13 & 0 & \\
\hline
\end{tabular}

Data are presented as numbers or means (standard deviations).

ETE: extrathyroidal extension, T, N stage: according to AJCC $8^{\text {th }}$ edition

no significant association between lymphocytic thyroiditis and the two molecular subtypes. We also compared RFS between the two clusters. There was no significant difference in RFS between the two groups (Supplementary Fig. 2). Overall, cluster 1 showed more frequent existence of nodular hyperplasia with indolent behavior whereas cluster 2 showed ag- gressive clinical behavior (Table 1).

The presence of BRAF ${ }^{\mathrm{V} 600 \mathrm{E}}$ mutation is one of the most influencing factor in tumor progression. Thus, multivariate analysis using stepwise logistic regression was conducted on parameters shown to be significant in univariate analysis to identify the role of cluster 2 as an independent predictor of the aggressive pheno- 
types of multifocal PTC (Table 2). Results of multivariate analysis revealed that cluster 2 was an independent risk factor for lymph node metastasis ( $p$ $<0.0001$, OR: 4.464) and the presence of $\mathrm{BRAF}^{\mathrm{V} 600 \mathrm{E}}$ mutation ( $p<0.0001$, OR: 5.411). Nodular hyperplasia was also a significantly related factor between two groups ( $p=0.035$, OR: 0.456) (Table 2).

\section{Comparison of Clinicopathologic Parameters between Cluster 1 and Cluster 2 Patients without BRAF Mutation and RAS Mutation}

Previous studies have reported that BRAFV600E mutated multifocal PTCs have aggressive phenotype. ${ }^{19,20)}$ Consistent with previous results, the pres-

Table 2. Multivariate analysis of clinicopathologic factors between cluster 1 and cluster 2

\begin{tabular}{lcccc}
\multicolumn{1}{c}{ Factors } & $\operatorname{Exp}(\beta)$ & $\mathrm{SE}$ & $95.0 \% \mathrm{Cl}$ & $\mathrm{p}$-value \\
\hline Tumor size & 0.964 & 0.570 & $(0.315,2.948)$ & 0.948 \\
Minor+ moderate/advanced ETE & 1.695 & 0.454 & $(0.696,4.131)$ & 0.245 \\
LN metastasis & 4.464 & 0.427 & $(1.932,10.313)$ & $<0.0001$ \\
Nodular hyperplasia & 0.383 & 0.456 & $(0.157,0.935)$ & 0.035 \\
BRAFV00E mutation & 5.411 & 0.435 & $(2.307,12.694)$ & $<0.0001$ \\
RAS mutation & 0.531 & 0.144 & $(0.387,0.675)$ & $<0.0001$ \\
\hline
\end{tabular}

Data analyzed using a stepwise logistic.

$\mathrm{Cl}$ : confidence interval, ETE: extrathyroidal extension, $\operatorname{Exp}(\beta)$ : odds ratio, SE:standard error

Table 3. Case distribution according to cluster 1 and cluster 2 in patients without BRAF or RAS mutation $(n=95)$

\begin{tabular}{|c|c|c|c|}
\hline & \multicolumn{2}{|c|}{ Cluster } & \multirow{2}{*}{$p$-value } \\
\hline & Cluster $1 \quad(n=51)$ & Cluster $2(n=44)$ & \\
\hline Age & & & 0.299 \\
\hline Mean & $50.9(12.1)$ & $47.6(10.3)$ & \\
\hline Gender & & & 0.800 \\
\hline Male & 15 & 14 & \\
\hline Female & 36 & 30 & \\
\hline Size $(\mathrm{cm})$ & & & 0.602 \\
\hline Mean diameter & $1.81(1.2)$ & $1.50(1.1)$ & \\
\hline ETE & & & 0.488 \\
\hline None & 40 & 30 & \\
\hline Minimal & 10 & 12 & \\
\hline Moderate/Advanced & 1 & 2 & \\
\hline T stage & & & 0.370 \\
\hline T1 & 34 & 33 & \\
\hline $\mathrm{T} 2$ & 14 & 7 & \\
\hline T3 & 3 & 4 & \\
\hline$N$ stage & & & $<0.001$ \\
\hline NO & 39 & 12 & \\
\hline $\mathrm{N} 1 \mathrm{a}$ & 6 & 14 & \\
\hline N1b & 6 & 18 & \\
\hline Stage & & & 0.096 \\
\hline Stage I & 43 & 31 & \\
\hline Stage II & 8 & 10 & \\
\hline Stage III & 0 & 3 & \\
\hline Unilateral/Bilateral & & & 0.141 \\
\hline Unilateral & 36 & 24 & \\
\hline Bilateral & 15 & 20 & \\
\hline
\end{tabular}

Data are presented as numbers or means (standard deviations).

ETE: extrathyroidal extension, TNM classification: according to AJCC $8^{\text {th }}$ edition 
ence of BRAFV600E mutation was significantly related to our classification using integrated genomic analysis. However, some patients with BRAF ${ }^{\mathrm{V} 600 \mathrm{E}}$ mutation included in cluster 1 group showed indolent clinical behavior. This suggests that there is another molecular mechanism independent of mutation of $\mathrm{BRAF}^{\mathrm{V}}{ }^{\mathrm{E}} \mathrm{COE}$ in multifocal PTCs that is associated with tumor progression. After excluding patients with BRAF mutation ( $n=114)$, and RAS mutation ( $n=21)$, we re-analyzed clinicopathologic findings between cluster 1 $(n=51)$ and cluster $2(n=44)$ (Table 3). Interestingly, presence of lymph node metastasis was significantly associated with cluster 2 group, compared to cluster 1 group (Table 3). These finding support that our classification using integrated genomic analysis may have potential to determine the prognosis of multifocal PTCs, independent of BRAF or RAS mutation status.

\section{Selection of Specific Gene Signature in Each Group}

Next, we tried to select genes differentially expressed between cluster 1 and cluster 2 multifocal

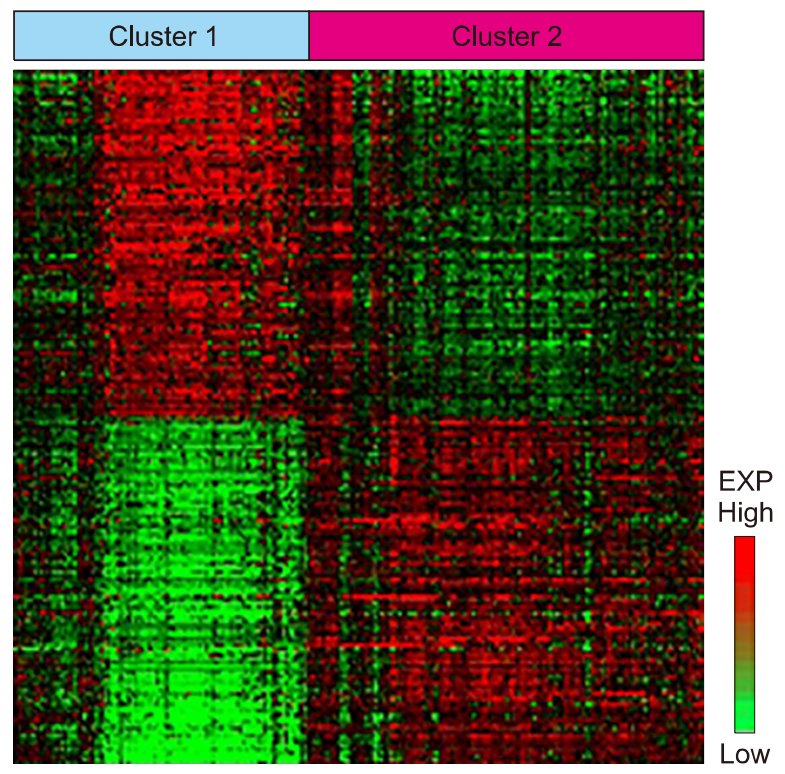

Fig. 3. Each cluster-specific gene expression patterns shown in heatmap. Data given in the matrix represent the expression level of a gene feature in an individual patient. We selected 100 group-specific genes to draw the heatmap. Red and green colors in cell indicate relative high and low expression, respectively.
PTCs. We applied stringent cutoff criteria $(p<0.001$, Student t-test; and 2-fold difference) and identified 100 genes for each group (Supplementary Data 2). In Fig. 3, data given in the matrix represent expression level of a gene feature in an individual patient. We selected 100 genes that were cluster specific and drew a heatmap. Red and green colors in the heatmap reflected relative high and low expression, respectively.

\section{Significant Canonical Signaling Pathways Enriched in Cluster 2 Revealed Aggressive Behavior of Multifocal PTC Using Ingenuity Pathway Analysis (IPA)}

IPA revealed that each cluster had different canonical signaling pathways (Supplementary Data 3). Diverse immune related pathways such as dendritic cell maturation, Th1 pathway, IL-6 pathway, and Leukocyte Extravasation signaling were enriched in cluster 2. P-value and ratio are presented in Table 4.

\section{Discussion}

Using TCGA mRNA expression data, we found that multifocal PTC had two distinct molecular subtypes for the first time. These two subgroups using molecular classification revealed significant different clinical behaviors and different molecular pathways. Multifocal PTC in cluster 1 showed indolent clinical behavior whereas multifocal PTC in cluster 2 showed aggressive clinical behavior, suggesting the importance of integrated genomic analysis for tumor aggressiveness in multifocal PTC. Our study could provide reasons why prognostic effects on tumor progression in multifocality are controversial.

Multifocality is frequently observed in patients with PTC. It is emerging as a prognostic marker in terms of lymph node metastasis and regional recurrence. ${ }^{3,6)}$ However, the prognostic significance of multifocality remains controversial. Moreover, there is no exact biomarker to predict the prognosis of multifocal PTC. Some groups have focused on the importance of bilateral PTC rather than unilateral multifocal PTC in tumor progression of multifocal PTC. However, prognostic effects of bilateral PTC are also controversial. 
Genomic Classifications in Multifocality

Table 4. Altered canonical pathways in cluster 2

\begin{tabular}{lcc}
\multicolumn{1}{c}{ Ingenuity canonical pathways } & - log ( $p$-value $)$ & Ratio \\
\hline IL-6 signaling & 1.38 & 0.0469 \\
Dendritic cell maturation & 3.9 & 0.0674 \\
Th1 pathway & 4.12 & 0.0815 \\
Th2 pathway & 5.05 & 0.0867 \\
iCOS-iCOSL signaling in T helper cells & 1.96 & 0.0569 \\
Role of NFAT in regulation of the immune response & 0.79 & 0.0323 \\
Leukocyte extravasation signaling & 2.99 & 0.0569 \\
PKC $\theta$ signaling in T lymphocytes & 1.42 & 0.044 \\
NF- $\kappa$ B signaling & 0.544 & 0.0276 \\
Signaling by Rho family GTPases & 0.255 & 0.0198 \\
Intrinsic prothrombin activation pathway & 5.86 & 0.19 \\
Calcium-induced T lymphocyte apoptosis & 1.39 & 0.0606 \\
B cell receptor signaling & 0.283 & 0.0209 \\
IL-8 signaling & 0.263 & 0.0203 \\
\hline
\end{tabular}

Previous study has suggested that patients with bilateral PTCs show more advance tumor stage, lymph node metastasis, and higher rates of ETE and BRAF $^{\mathrm{V} 600 \mathrm{E}}$ mutation than those with unilateral-multifocal PTCs. ${ }^{6)}$ Other study has indicated that bilateral and multifocal PTCs have aggressive pathologic features. ${ }^{3)}$ However, only multifocality is significantly associated with an increased risk of disease recurrence. ${ }^{3)}$ Therefore, tumor location is insufficient as a predictive biomarker to treat multifocal PTC. Here, we investigated tumor location of unilateral PTC and bilateral PTC in cluster 1 and cluster 2 using molecular classification (Table 1). We found no significant difference in the proportion of bilateral PTC between the two groups. These results support that molecular classification using integrated genomic analysis may be useful for predicting tumor aggressiveness rather than the location of tumor in multifocal PTC.

Previous studies about papillary microcarcinoma have found that recurrence is significantly higher in the presence of multifocality. ${ }^{21-23)}$ However, the effect of multifocality in PTC with tumor size larger than $1 \mathrm{~cm}$ is rarely reported. A recent meta-analysis about multifocal PTC has shown that there is no significant association between tumor size and multifocality, although diverse clinic-pathologic parameters including lymph node metastasis, ETE, and disease recurrence are associated with multifocality. ${ }^{2)}$ In this study, we analyzed all multifocal PTC, including microcarcinoma and mac- rocarcinoma, from TCGA database to evaluate the importance of tumor size in determining tumor progression in multifocal PTCs. Our results are consistent with previous meta-analysis. ${ }^{1)}$ Tumor size in cluster 2 showing aggressive behavior was significantly smaller than that in cluster 1 showing indolent behavior (Table 1). Additionally, in multivariate analysis (Table 2), there was no significant association between tumor size $(>1$ $\mathrm{cm}$ ) and molecular classification, although lymph node metastasis and $\mathrm{BRAF}^{\mathrm{V} 600 \mathrm{E}}$ mutation were significantly more frequent in cluster 2 compared to those in cluster 1. Thus, tumor size was not a major influencing factor of tumor behavior in multifocal PTC.

Since most studies have focused on the association of clinicopathologic parameters with tumor progression of multifocal PTC without performing molecular analysis, the mechanism involved in the aggressive behavior of multifocal PTC has remains unclear. A recent study has suggested that BRAF ${ }^{\mathrm{V} 600 \mathrm{E}}$ mutation, the most well-known mutation related to thyroid cancer prognosis, is significantly associated with tumor progression in multifocal PTC. ${ }^{24)}$ However, several recent studies have failed to corroborate such findings. ${ }^{25,26)}$ Thus, the significance of BRAF ${ }^{\mathrm{V} 600 \mathrm{E}}$ mutations in multifocal PTC remains debatable. We analyzed BRAF ${ }^{\mathrm{V} 600 \mathrm{E}}$ mutation status between the two clusters to evaluate the effect of BRAF ${ }^{\mathrm{V} 600 \mathrm{E}}$ mutation through molecular classifications. Cluster 2 showed significant higher existence of $\mathrm{BRAF}^{\mathrm{V} 600 \mathrm{E}}$ mutation and more aggressive 
clinical behaviors compared to cluster 1. Currently, the presence of BRAF ${ }^{\mathrm{V} 600 \mathrm{E}}$ mutation was significantly related to our classification using integrated genomic analysis based on multivariate analysis (Table 2). However, some participants with BRAF ${ }^{\mathrm{V} 600 \mathrm{E}}$ mutation were included in cluster 1 with indolent clinical behavior. These results are inconsistent with findings of our previous report. ${ }^{27)}$ When we analyzed TCGA database from all patients with PTC using unsupervised clustering, there were two distinct mRNA expression patterns and all BRAF ${ }^{\mathrm{V} 600 \mathrm{E}}$ mutated PTCs were included in one cluster group. ${ }^{27)}$ Molecular mechanisms involved in tumor behavior of multifocal PTCs might be different from those involved in unifocal PTCs. We also investigated clinicopathologic parameters of BRAF ${ }^{\text {wild }}$ multifocal PTCs in cluster 1 , different from BRAF ${ }^{\text {wild }}$ multifocal PTCs in cluster 2, to determine whether our molecular classification using integrated gene-level approach could be used as a surrogate tool to predict prognosis in multifocal PTC independent on BRAF ${ }^{\mathrm{V} 600 \mathrm{E}}$ mutation. Interestingly, BRAF ${ }^{\text {wild }}$ multifocal PTCs in cluster 1 showed indolent clinical behavior whereas BRAF ${ }^{\text {wild }}$ multifocal PTCs in cluster 2 showed aggressive behavior (Table 3), suggesting the prognostic possibility using our molecular classification.

We also analyzed somatic mutation data between the two groups. Multifocal PTC in cluster 1 had frequent RAS family mutations, including NRAS, KRA, and HRAS. In contrast, multifocal PTCs in cluster 2 did not have any RAS mutation. RAS mutation in BRAF ${ }^{\text {wild }}$ PTCs is known to be associated with a lower risk of tumor progression. ${ }^{28-30)}$ These results are similar to those of our previous study about BRAF ${ }^{\text {wild }}$ PTC. ${ }^{27)}$ However, the prevalence of RAS family was detected in only $11 \%$ of our data. We also selected genes differentially expressed between cluster 1 and cluster 2 to search for upstream or downstream of functionally important genes. Comparison of differentially expressed genes between the two clusters revealed that there was no different difference in expression of well-known genes such as BRAF, TERT, RAS, RET, or TP53. Interestingly, when we analyzed prominent molecular pathway in cluster 2 compared to that in cluster 1, IPA analysis revealed that many immune-related signaling including IL-6 signaling and macrophage signaling were related to tumor aggressiveness (Table 4). IL-6 is frequently viewed as a pro-inflammatory cytokine with functions similar to tumor necrosis factor (TNF) and $\mathrm{IL}-1 \mathrm{~b}$ in the context of inflammation. ${ }^{31)}$ During tumor progression, classically activated macrophages or M1- macrophages are characterized by high expression of IL-6, TNF, and iNOS as well as a dendritic cell-like phenotype to tumor progression, and IL-6 is known to have detrimental effects. Its action is frequently linked to tumor growth, progression, and relapse. ${ }^{32)}$ Cluster 2 with aggressive behavior in our molecular classification showed upregulated dendritic cell maturation signaling, production of $\mathrm{NO}$ and reactive oxygen species in macrophages, Th1 Pathway, and Th2 Pathway, as well as upregulation of IL-6 signaling axis. These findings suggest that IL -6 signaling axis has an important role in thyroid cancer progression. Thus, targeting IL-6 signaling might contribute to clinical management of multifocal PTC patients.

Previous findings have suggested that the pathogenesis of multifocal PTC involves intraglandular spread of a single clone or from independent progenitor clones. ${ }^{33-36)}$ The exact prognostic value of independent clonal origin and intra-thyroidal metastases from a single malignant focus is unknown. ${ }^{36)}$ Currently, it remains inconclusive whether each tumor in multifocal PTC occurs independently or spreads from a single tumor mass in the thyroid gland in our study because TCGA database have gene analysis of single tumor in each patient. To clarify the importance of clonal origin in determining molecular subtypes, a large scaled study using whole genome sequencing of all tumor samples of multifocal PTCs will be necessary. This study identified that multifocal PTC has two distinct molecular subtypes with distinctive clinical behaviors. Using these molecular subtypes, we can predict tumor aggressiveness and make an appropriate treatment plan of multifocal PTC.

In conclusion, multifocal PTC has two distinct molecular subtypes with distinctive clinical behaviors, independently the presence of BRAF or RAS mutation. Our data suggested the clinical implications of the transcriptomic signature to predict clinical outcomes of 
multifocal PTC.

\section{Acknowledgments}

We appreciated patients and their families who generously donated their tissues to TCGA, as well as the members of TCGA who collected and disclosed valuable data. This research was supported by Hallym University Research Fund 2018 (H20180239), and was supported by the Basic Science Research Program, National Research Foundation, Ministry of Science and ICT, Future Planning, Korea (NRF-2017R1D1A1B0302 7820).

\section{Conflicts of Interest}

No potential conflict of interest relevant to this article was reported.

\section{Orcid}

Yea Eun Kang: https://orcid.org/0000-0002-2012-3716 Boyoung Hwang: https://orcid.org/0000-0003-0230-836X Ju Hee Lee: https://orcid.org/0000-0001-5976-7175 Minho Shong: https://orcid.org/0000-0002-0247-7115 Hyon-Seung Yi: https://orcid.org/0000-0002-3767-1954 Bon Seok Koo: https://orcid.org/0000-0002-5928-0006 Dong Jin Lee: https://orcid.org/0000-0002-2661-3361

\section{References}

1) Hundahl SA, Fleming ID, Fremgen AM, Menck HR. $A$ National Cancer Data Base report on 53,856 cases of thyroid carcinoma treated in the U.S., 1985-1995 [see commetns]. Cancer 1998;83(12):2638-48.

2) Joseph KR, Edirimanne S, Eslick GD. Multifocality as a prognostic factor in thyroid cancer: A meta-analysis. Int J Surg 2018;50:121-5.

3) Kim HJ, Sohn SY, Jang HW, Kim SW, Chung JH. Multifocality, but not bilaterality, is a predictor of disease recurrence/persistence of papillary thyroid carcinoma. World $J$ Surg 2013;37(2):376-84.

4) Park SY, Park YJ, Lee YJ, Lee HS, Choi SH, Choe G, et al. Analysis of differential BRAF(V600E) mutational status in multifocal papillary thyroid carcinoma: evidence of independent clonal origin in distinct tumor foci. Cancer 2006;107(8):1831-8.

5) Iacobone M, Jansson S, Barczynski M, Goretzki P. Multifocal papillary thyroid carcinoma--a consensus report of the European
Society of Endocrine Surgeons (ESES). Langenbecks Arch Surg 2014;399(2):141-54.

6) Wang W, Su X, He K, Wang Y, Wang H, Wang H, et al. Comparison of the clinicopathologic features and prognosis of bilateral versus unilateral multifocal papillary thyroid cancer: An updated study with more than 2000 consecutive patients. Cancer 2016;122(2):198-206.

7) Amin A, Younis G, Sayed K, Saeed Z. Cervical lymph node metastasis in differentiated thyroid carcinoma: does it have an impact on disease-related morbid events? Nucl Med Commun 2015;36(2):120-4.

8) Blanchard C, Brient C, Volteau C, Sebag F, Roy M, Drui $\mathrm{D}$, et al. Factors predictive of lymph node metastasis in the follicular variant of papillary thyroid carcinoma. Br J Surg 2013;100(10):1312-7.

9) Pinyi Z, Bin Z, Jianlong B, Yao L, Weifeng Z. Risk factors and clinical indication of metastasis to lymph nodes posterior to right recurrent laryngeal nerve in papillary thyroid carcinoma: a single-center study in China. Head Neck 2014;36(9):1335-42.

10) Singhal S, Sippel RS, Chen H, Schneider DF. Distinguishing classical papillary thyroid microcancers from follicular-variant microcancers. J Surg Res 2014;190(1):151-6.

11) Konturek A, Barczynski M, Nowak W, Richter P. Prognostic factors in differentiated thyroid cancer-a 20-year surgical outcome study. Langenbecks Arch Surg 2012;397(5):809-15.

12) Ahn D, Sohn JH, Kim JH, Shin CM, Jeon JH, Park JY. Preoperative subclinical hypothyroidism in patients with papillary thyroid carcinoma. Am J Otolaryngol 2013;34(4):312-9.

13) Cancer Genome Atlas Research Network. Integrated genomic characterization of papillary thyroid carcinoma. Cell 2014; 159(3):676-90.

14) Simon R, Lam A, Li MC, Ngan M, Menenzes S, Zhao Y. Analysis of gene expression data using BRB-ArrayTools. Cancer Inform 2007;3:11-7.

15) Eisen MB, Spellman PT, Brown PO, Botstein D. Cluster analysis and display of genome-wide expression patterns. Proc Natl Acad Sci U S A 1998;95(25):14863-8.

16) Kim SJ, Myong JP, Jee HG, Chai YJ, Choi JY, Min HS, et al. Combined effect of Hashimoto's thyroiditis and BRAF(V600E) mutation status on aggressiveness in papillary thyroid cancer. Head Neck 2016;38(1):95-101.

17) Bircan HY, Koc B, Akarsu C, Demiralay E, Demirag A, Adas $\mathrm{M}$, et al. Is Hashimoto's thyroiditis a prognostic factor for thyroid papillary microcarcinoma? Eur Rev Med Pharmacol Sci 2014;18(13):1910-5.

18) Lee YK, Park KH, Park SH, Kim KJ, Shin DY, Nam KH, et al. Association between diffuse lymphocytic infiltration and papillary thyroid cancer aggressiveness according to the presence of thyroid peroxidase antibody and BRAF(V600E) mutation. Head Neck 2018;40(10):2271-9.

19) Qu HJ, Qu XY, Hu Z, Lin Y, Wang JR, Zheng CF, et al. The synergic effect of BRAF(V600E) mutation and multifocality on central lymph node metastasis in unilateral papillary thyroid carcinoma. Endocr J 2018;65(1):113-20.

20) Kimbrell HZ, Sholl AB, Ratnayaka S, Japa S, Lacey M, Carpio G, et al. BRAF testing in multifocal papillary thyroid carcinoma. Biomed Res Int 2015;2015:486391. 
21) Giordano D, Gradoni P, Oretti G, Molina E, Ferri T. Treatment and prognostic factors of papillary thyroid microcarcinoma. Clin Otolaryngol 2010;35(2):118-24.

22) Hay ID, Hutchinson ME, Gonzalez-Losada T, McIver B, Reinalda ME, Grant CS, et al. Papillary thyroid microcarcinoma: a study of 900 cases observed in a 60-year period. Surgery 2008;144(6):980-7; discussion 7-8.

23) Lombardi CP, Bellantone R, De Crea C, Paladino NC, Fadda G, Salvatori M, et al. Papillary thyroid microcarcinoma: extrathyroidal extension, lymph node metastases, and risk factors for recurrence in a high prevalence of goiter area. World J Surg 2010;34(6):1214-21.

24) Xing M, Alzahrani AS, Carson KA, Viola D, Elisei R, Bendlova B, et al. Association between BRAF V600E mutation and mortality in patients with papillary thyroid cancer. JAMA 2013;309(14):1493-501.

25) Henke LE, Pfeifer JD, Ma C, Perkins SM, DeWees T, El-Mofty $\mathrm{S}$, et al. BRAF mutation is not predictive of long-term outcome in papillary thyroid carcinoma. Cancer Med 2015;4(6): 791-9.

26) Gouveia C, Can NT, Bostrom A, Grenert JP, van Zante A, Orloff LA. Lack of association of BRAF mutation with negative prognostic indicators in papillary thyroid carcinoma: the University of California, San Francisco, experience. JAMA Otolaryngol Head Neck Surg 2013;139(11):1164-70.

27) Kim HK, Lee I, Lee J, Chang HS, Soh EY, Park IS, et al. $B R A F($ wild) papillary thyroid carcinoma has two distinct $m R N A$ expression patterns with different clinical behaviors. Head Neck 2018;40(8):1707-18.

28) Song YS, Lim JA, Park YJ. Mutation profile of well-differentiated thyroid cancer in Asians. Endocrinol Metab (Seoul) 2015;30(3): 252-62.

29) An JH, Song KH, Kim SK, Park KS, Yoo YB, Yang JH, et al. RAS mutations in indeterminate thyroid nodules are predictive of the follicular variant of papillary thyroid carcinoma. Clin Endocrinol (Oxf) 2015;82(5):760-6.

30) Oh KH, Jung KY, Baek SK, Woo JS, Cho JG, Kwon SY. Relation between RASSF1A methylation and BRAF mutation in thyroid tumor. Int J Thyroidol 2017;11(2):123-9.

31) Mauer J, Denson JL, Bruning JC. Versatile functions for IL-6 in metabolism and cancer. Trends Immunol 2015;36(2):92-101.

32) Krawczyk CM, Holowka T, Sun J, Blagih J, Amiel E, DeBerardinis RJ, et al. Toll-like receptor-induced changes in glycolytic metabolism regulate dendritic cell activation. Blood 2010;115(23):4742-9.

33) Jovanovic L, Delahunt B, McIver B, Eberhardt NL, Grebe SK. Most multifocal papillary thyroid carcinomas acquire genetic and morphotype diversity through subclonal evolution following the intra-glandular spread of the initial neoplastic clone. J Pathol 2008;215(2):145-54.

34) Lin X, Finkelstein SD, Zhu B, Silverman JF. Molecular analysis of multifocal papillary thyroid carcinoma. J Mol Endocrinol 2008;41(4):195-203.

35) McCarthy RP, Wang M, Jones TD, Strate RW, Cheng L. Molecular evidence for the same clonal origin of multifocal papillary thyroid carcinomas. Clin Cancer Res 2006;12(8): 2414-8.

36) Lu Z, Sheng J, Zhang Y, Deng J, Li Y, Lu A, et al. Clonality analysis of multifocal papillary thyroid carcinoma by using genetic profiles. J Pathol 2016;239(1):72-83. 


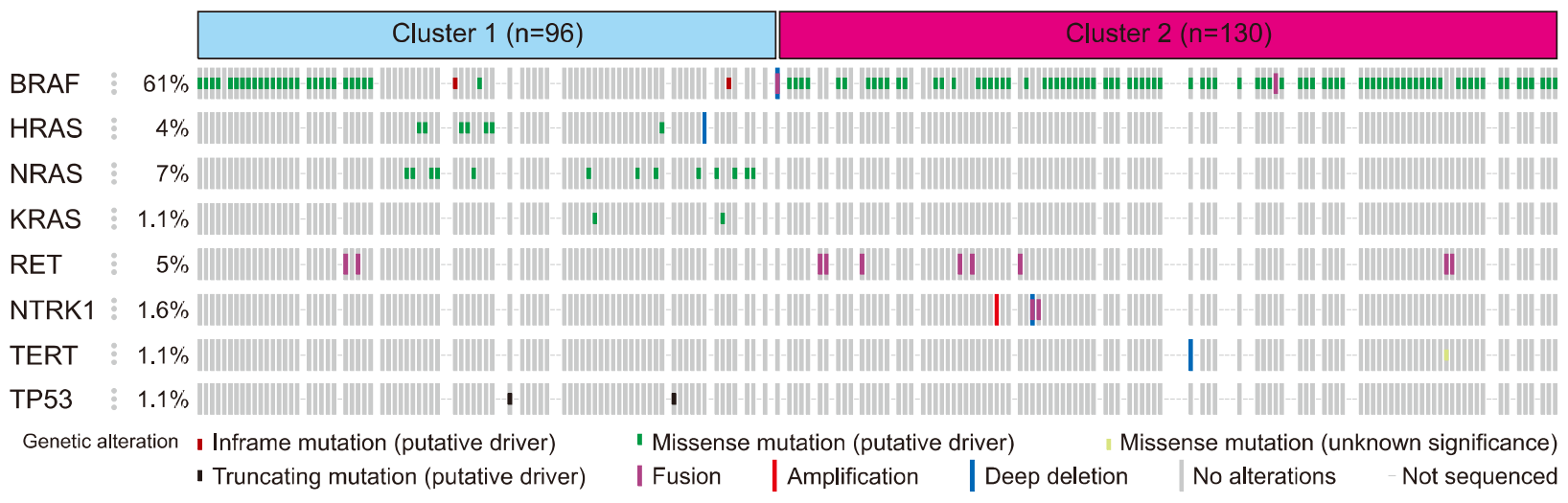

Supplementary Fig. 1. Comparison of mutation profiles between cluster 1 and cluster 2 .

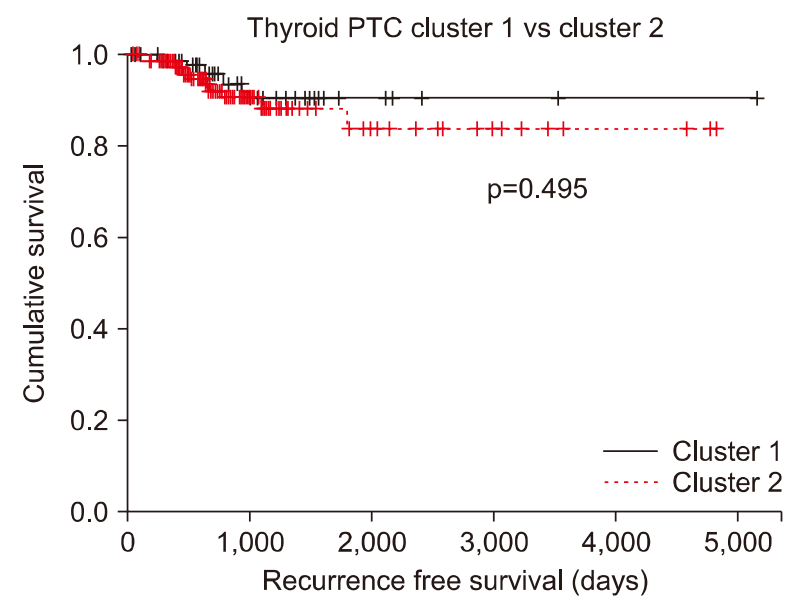

Supplementary Fig. 2. Comparison of reurrence free survival curve between cluster 1 and cluster 2 . 